02

\title{
Atomic Structure and Optical Properties of Plasma Enhanced Chemical Vapor Deposited SiCOH Low- $k$ Dielectric Film*
}

(c) V.N. Kruchinin ${ }^{1}$, V.A. Volodin ${ }^{1,2}$, S.V. Rykhlitskii ${ }^{1}$, V.A. Gritsenko ${ }^{1,2,3}$, I.P. Posvirin ${ }^{4}$, Xiaoping Shi ${ }^{5}$, and M.R. Baklanov ${ }^{6,7}$

${ }^{1}$ Rzhanov Institute of Semiconductor Physics, SB RAS,

630090 Novosibirsk, Russia

${ }^{2}$ Novosibirsk State University,

630090 Novosibirsk, Russia

${ }^{3}$ Novosibirsk State Technical University,

630073 Novosibirsk, Russia

${ }^{4}$ Boreskov Institute of Catalysis SB RAS,

630090 Novosibirsk, Russia

${ }^{5}$ Beijing Naura Microelectronics,

E-Town, Beijing, China

${ }^{6}$ North China University of Technology,

Beijing, China

${ }^{7}$ Russian Technological University MIREA,

Moscow, Russia

e-mail: vladd.kruch@yandex.ru

Received October 06, 2020

Revised December 03, 2020

Accepted December 26, 2020

The SiCOH low- $k$ dielectric film was grown on Si substrate using plasma-enhanced chemical vapor deposition method. Atomic structure and optical properties of the film were studied with the use of X-ray photoelectron spectroscopy (XPS), Fourier transform infrared (FTIR) absorption spectroscopy, Raman spectroscopy, and ellipsometry. Analysis of XPS data showed that the low- $k$ dielectric film consists of $\mathrm{Si}_{-} \mathrm{O}_{4}$ bonds $(83 \%)$ and $\mathrm{Si}-\mathrm{SiO}_{3}$ bonds $(17 \%)$. In FTIR spectra some red-shift of $\mathrm{Si}-\mathrm{O}-\mathrm{Si}$ valence (stretching) vibration mode frequency was observed in the low- $k$ dielectric film compared with the frequency of this mode in thermally grown $\mathrm{SiO}_{2}$ film. The peaks related to absorbance by $\mathrm{C}-\mathrm{H}$ bonds were observed in FTIR spectrum. According to Raman spectroscopy data, the film contained local $\mathrm{Si}-\mathrm{Si}$ bonds and also $\mathrm{C}-\mathrm{C}$ bonds in the $s-p^{3}$ and $s-p^{2}$ hybridized forms. Scanning laser ellipsometry data show that the film is quite homogeneous, homogeneity of thickness is $\sim 2.5 \%$, and homogeneity of refractive index is $\sim 2 \%$. According to the analysis of spectral ellipsometry data, the film is porous (porosity is about $24 \%$ ) and contains clusters of amorphous carbon $(\sim 7 \%)$.

Keywords: low- $k$ dielectrics, PECVD, optical properties, atomic structure.

* Полный текст статьи опубликован в „Optics and Spectroscopy“ 2021 V. 129. N 5. 